\title{
Multi-Criteria Gateway Selection \& Multipath Routing Protocol for Hybrid MANETs
}

\author{
Rajesh Gargi \\ Department of Computer \\ Science Engineering, IIET, \\ Kinana, Jind
}

\author{
Yogesh Chaba \\ Department of Computer \\ Science and Engineering, \\ GJUST, Hisar
}

\author{
R. B. Patel \\ Faculty, Department of IT \& \\ Computer Science Engineering, \\ DCRUST, Murthal
}

\begin{abstract}
In hybrid mobile ad hoc network (MANET), the mobility of the ad hoc nodes can cause route failures and necessitates the new route discovery. Also the mobility can result in delay and overhead. In this paper, we propose a multi-criteria gateway selection and multipath routing protocol for hybrid MANET that considers mobility metric as one of the criterion for the selecting the gateway. For gateway selection, a combined weight value is determined based on the metrics mobility, inter and intra MANET traffic load and residual energy using simple additive weighing (SAW) technique. Among the selected path from the multiple paths, the gateway with maximum weight is selected. If such a gateway does not exist, alternate path is selected from the multi path set. By simulation results, we show that our proposed protocol is more efficient technique for gateway selection.
\end{abstract}

\section{Keywords}

Mobile ad hoc network (MANET), simple additive weighing, Multipath Routing Protocol, mobile node (MN), gateways (GWs).

\section{INTRODUCTION}

\subsection{Routing in Mobile Ad hoc Network (MANET)}

A mobile ad hoc network (MANET) is a dynamic wireless network which has a free movement of nodes and arranges in a random manner [1]. Without the help of any pre-existing network infrastructure, the MANETs can be setup wherever and whenever necessary. Being an autonomous system, the mobile hosts act as routers and have a random movement [2]. Multihop routing is used for communication in every mobile node $(\mathrm{MN})$ in the MANET, since both the router and the user role is played by the MN. [3]. The dynamic nature of network topology and the resource constraints makes MANET routing a tedious process. Transmitting messages through wireless channels become a major problem due to link reliability. The minimum hop count routing selects path with less capacity rather the best paths that exist in the network and so good quality paths are not built using this routing [1]. On the basis of their reaction to topological changes, routing protocols are divided into proactive (table-driven), and reactive (on-demand). In general three issues need to be addressed in the routing protocol: route discovery, data forwarding, and route maintenance. [4]

\subsection{Hybrid MANET}

- Hybrid MANET is imparted by the gateways (GWs) connecting the MANET with the internet which also gives advanced communication, network scalability, and pervasive sustainable environments.

- Studies related to GW management, mobility management, addressing, and routing are undergone in the hybrid MANETs. Additionally, logical and technological developments are needed for robust interconnection [3].
- With the fixed IGWs the hybrid MANET provides internet access to the MANET nodes. It also exploits mobility capability of additional mobile nodes (mobile IGWs). The benefits of the proactive and reactive approaches are also balanced by the hybrid approach.

- The dynamic network topology leads to uncertainty in the connectivity of the mobile nodes with gateway nodes and mobile nodes with other active mobile nodes. In the local MANET, there is a delay in finding route to destination due to the mobility of mobile nodes. [5]

\subsection{Dynamic MANET On-demand (DYMO) Routing Protocol}

\subsubsection{DYMO Routing}

DYMO routing protocol is a reactive protocol developed for MANET. All the nodes between the source and destination exchange routing information through routing information accumulation [7]. Route discovery and route maintenance are the two operations of the DYMO routing protocol. The originate node, in routing discovery, multicasts a RREQ to all the nodes immediately. In order to review the freshness of the route request, the RREQ consists of a sequence number to enable other nodes. Until the request reaches the target node, the network will be flooded with the RREQs. The originating node receives an RREP which is unicast hop-by-hop from the target node. [6].

\subsubsection{Gateway Selection}

When a mobile node sends a data packet to fixed network, the packets are transmitted to the gateway which acts as a bridge between a MANET and the Internet. On receiving RREQ, the gateway cross checks with the routing table for destination IP address which has been précised in the RREQ message. If the address is not found, then gateway sends RREP_I flag to the originator, else it unicasts a normal RREP, but may also optionally send a RREP_I back to the originator of the RREQ. [8]

Proactive Gateway Discovery, Reactive Gateway Discovery, Hybrid Gateway Discovery, Adaptive Gateway Discovery, and Maximal Benefit Coverage are the various gateway discovery processes.

- Proactive Gateway Discovery: Gateway broadcasts a Gateway Advertisement message after each interval. Mobile nodes in the gateway's transmission range receive the advertisement and those without the route to the gateway, builds a route entry for it in their routing tables.

- Reactive Gateway Discovery: By performing expanding ring search, the node willing to communicate with the network will contact it within the ad hoc network. A new route is found towards the Internet, if there is no reply after the search.

- Hybrid Gateway Discovery: The TTL-limited messages are flooded by the gateways which will be forwarding only up to few hops away from the gateway. Proactive approach 
has been carried out by the sources within flooding area and outside that it acts as reactive.

- Adaptive Gateway Discovery: Information is easily provided by the gateway only if it is routing those datagrams that it would receive anyway. Also the number of hops of its active source location is maintained.

- Maximal Benefit Coverage: The overhead of flooding GWADV messages up to $t$ hops plus the overhead associated to the discovery of gateways by sources at distances longer than $t$ hops can be minimized by selecting a TTL t.

- When multiple nodes are discovered for internet access, the Internet gateway selection is used. We have to choose a metric for selecting the right gateway. [8]

\subsubsection{Issues in DYMO Routing}

The DYMO protocol is comparatively simpler than the AODV protocol but mostly the routing logic layer provides ease whereas the challenges and problems are valid for both DYMO and AODV. Some of the issues are

- Since the failed connection attempt is not registered, the routes may not be known in advance when on-demand ad hoc routing protocols are used. In order to discover a route to the destination, the routing node must be notified about the connection attempts. Additionally, when the route discovery is ongoing, the packets must be buffered.

- In the current network stack architecture, the main problem is that, only after the packet crosses the boundary between the user space and the kernel space, we bother about the need for the route. So when to initiate the route discovery is not known.

- During route discovery, when and how to buffer packets: Packets must be buffered while route recovery is active if the packets are destined to hosts with unknown destination. The packets must be reinserted into the IP layer in case the route is found and then sent to the destination. The packet should be discarded in case the route is not found and then the application program should be reported.

- If a valid route does not exist then when to create an RERR: For a packet if no valid route table entry exists, the IP layer discards it under normal condition. Then an ICMP destination host unreachable message is returned. As an alternate, the notification is sent to the routing node about the event.

- Available routing table information within DYMO nodes is not applicable when communication between nodes breaks from repositioning of a node(s). The performance against the conventional AODV algorithm becomes poorer since the DYMO's advantage of routing path accumulation is spurned by the increase in RREQ message size from RREQ accumulation. [7]

- The speed of simulation in large scale networks are affected by the higher end to end delays of the DYMO protocol. [10].

- In the DYMO approach, the mitigation of traffic concentration on a special-gateway s-GW and the MNs around them is not done properly. [3]

- The essential timeout mechanism and link monitoring for detecting broken links are not envisioned. There is a need to consider the process for reducing the number of cases in the state space analysis which are not actually taken into account. Hence state space analysis doesn't consider the route error processing, route maintenance, and dynamic topologies [6]

\subsection{Mobility in Hybrid MANET}

At the time of access to internet, for guaranteeing the characteristics such as self-configured, infrastructure-less and mobility controlled nature, various functions must be offered. The functions are as follows.

1) Determination of nodes position of MANET.

2) Discovery, selection and forwarding policy of the internet gateway (IGW).

3) Auto-configuration addressing technique.

4) Handoff control [11]

Typically, the mobility of the internet hosts is inside the similar broadcast field of internet gateway which is termed as 1-hop internet mobility management. The recent technologies utilize MANET as the assess network for the Internet in which MANETs either wrap the unfilled field or broadens the network from 1-hop to multihop in the existing access technologies which includes wireless LANs or cellular networks. Usually the link among a MANET node and an internet gateway (IGW) is multihop. Hence in general, there is no direct wireless connection between MANET node and IGW. As an alternative they are linked through other intermediate nodes. [17]

\subsection{Simple Additive Weighing (SAW)}

The simple additive weighing (SAW) technique corresponds to the multi-criteria decision making which is utilized to compute the metrics. The merit of this approach is that it relates multiple metrics that includes weight of significant level, score estimation, and gateway outranking depending on the user precedence.

Fundamentally SAW computes the complete grade of a metric as the weighted sum of all metric values. The steps involved in SAW are as follows.

1) Similar value are scaled

2) Weighting factors are applied.

3) Totaling the weight values of every metric.

\subsection{Proposed Work}

In paper [18], an efficient multi-path extension to DYMO with a load balancing technique for gateway selection is proposed. For gateway selection, a combined weight value is determined based on the metrics shortest distance, inter and intra MANET traffic load. Among the selected path from the multiple paths, the gateway with minimum weight is selected. If such a gateway does not exist, alternate path is selected from the multi path set.

The metric chosen for selection of the internet gateway (IGW) balances the traffic load alone and does not consider the mobility criteria. The weight computation is also performed in the destabilized manner.

But the mobility of ad hoc networks can cause the following issues.

At the time of mobility of ad-hoc nodes within a MANET domain, the issues such as inconsistent context and cascading effect occurs in the existence of multiple IGWs.

- During the communication among nodes, there may be a necessity that the packets should pass through several hops. As the intermediate node may be mobile in nature, node mobility can result in recurrent link failures and staleness of routes.

- Consequently there is occurrence of route errors and initiates a new route discovery process.

- The mobility can also result in overhead and delay. 
In order to overcome the above drawbacks, and also to make weight computation more accurate, in this paper, we propose a Multi-path Dymo routing protocol with Multi criteria based Gateway selection for Hybrid MANET.

\section{RELATED WORK}

Rakesh Kumar et al [12] initially investigated the load-aware routing protocols in MANET and depending on this analysis, a proactive load-aware gateway discovery scheme is proposed that considers the size of interface queue along with the traditional min hop metric. Though this approach permits an efficient handoff from one internet gateway, it upholds a seamless connectivity to a fixed host.

P. Venkateswaran et al [13] presented a MAC protocol with cluster head and gateway selection algorithm. It is predicted that clustering technique attains improved scalability as majority topology modifications inside the cluster are secreted from the remaining network. The gateway nodes are selected for inter-cluster communication. But they guarantee that the maximum number of clusters that are linked with the assistance of the single gateway does not go above its allowable overhead during the gateway selection process.

Quan Le-Trung et al [14] proposed a hybrid metric for choosing IGW for balancing the intra/inter-MANET traffic load between multiple IGWs on the similar MANET domain. This approach takes three components into consideration such as: Euclidean distance, load balance of inter-MANET traffic and load balance of intra-MANET traffic. The drawback of this technique is that it does not consider MANET's node location along with the utilization of the location based ad hoc routing.

Amandeep Kaur Saggu et al [15] introduced the Interface Queue Occupancy Algorithm and Internet Gateway Discovery Method for selecting the gateway. The purpose of gateway is to offer the internet connectivity. The level of congestion at each node is verified. Using the proposed approach, the issue of traffic in DSDV and congestion level is resolved.

Khaleel Ur Rahman Khan et al [16] proposed an efficient DSDV protocol for offering the bidirectional connectivity among ad hoc nodes and the hosts in the infrastructure-based networks. The proposed approach utilizes ad hoc hosts called mobile internet gateway to perform as a link among the two networks.

\section{PROPOSED WORK}

\subsection{Overview}

We propose a multi-criteria gateway selection and multipath routing protocol for Hybrid MANETs. The multiple paths are established using the dynamic MANET on-demand (DYMO) routing protocol. For gateway selection, a combined weight value is determined based on the metrics mobility, inter and intra MANET traffic load and residual energy using simple additive weighing (SAW) technique. As the scaling approach, this technique uses the positive and negative criteria so the metrics turns out to be a non-dimensional value. Following the normalization of the every metric to the equivalent scale, the weighting factor is determined using direct specification methodology. Then the overall weight value for each gateway candidate is computed by multiplying the weighting factors and normalized metric values and further summing of all these value. Among the selected path from the multiple paths, the gateway with maximum weight is selected. If such a gateway does not exist, alternate path is selected from the multi path set.

\subsection{Multi-path Extension to DYMO}

A technique for multi-path selection is proposed in hybrid MANETs so that even if one path fails the data can be routed through another path.

In the multi-path route discovery process, when several route replies arrive to the source from different nodes and path identifiers, the DYMO agent stores these nodes as next hops in the destination entry of its route table. Cut off problem is solved in easy way. In route request phase, the intermediate node registers all the paths with different last hops for sending RREP to the corresponding RREQ though they may arrive from the same neighbor.

We describe the request and the reply phase with the help of a figure1 and figure2. Figure 1 shows the request phase and Figure 2 shows the reply phase where nodes $\mathrm{X}$ and Y saves two paths with destination as $\mathrm{S}$ and the next hop.

$>$ The destination node receives the route request in the reply process and sends back the reply through the neighboring node from which it received the packet. The last hop value is same as the value in the request packet. The initial path used by the intermediate node with this last hop is the valid one to determine the next hop and other paths are detached though it has different last hop.

$>$ Suppose that, first of all, node D receives the route request from $X$ with last hop L. D sends a route reply with last hop $\mathrm{L}$ to $\mathrm{X}$ (RREP-L).Although $\mathrm{D}$ receives another route request from $X$, with a different last hop (in this case $M$ ), $\mathrm{D}$ discards the packet and it does not record this path.

> Similarly, if D hears a request from another node with last hop L, it obviously discards the packet too. Only if D receives a request from another node $(\mathrm{Y})$ with different last hop (M), does it save this path and send a new reply (RREP-M). When node $\mathrm{X}$ receives RREP-L it searches the path to node $S$ with last hop $\mathrm{L}$ and removes other paths with the same next hop as the selected path (e.g., it would remove the second row of the table in figure 2). Node Y removes the first row when it processes the RREP-M. This way we solve the route cutoff problem.

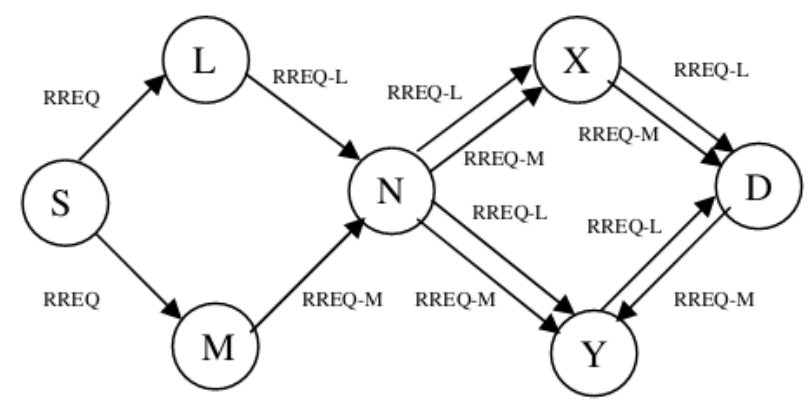

Figure 1: Request Phase

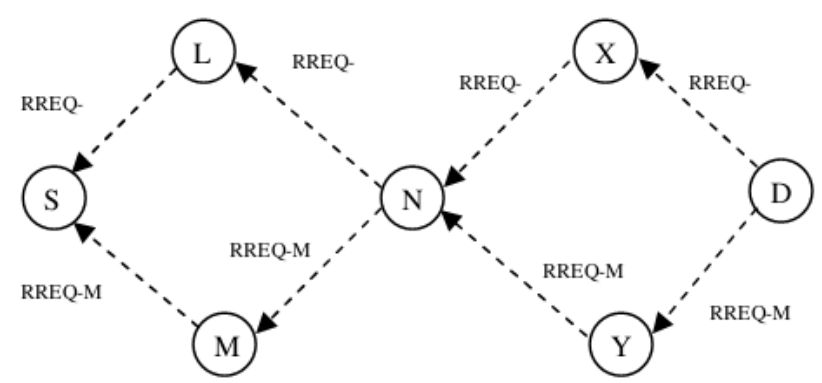

Figure 2: Reply Phase 
Each node has one or more routes for every possible destination, after the route discovery process. So, it must decide how to select them. The node chooses the route with lowest timeout value for every data packet. Timeout of this route is updated so that it becomes the route with largest timeout value. Then route with lowest timeout becomes different one and cyclically the routes are selected. [11].

\subsection{Parameters to Compute Weight Value}

The parameters involved in the computation of the weight value are as follows.

- The relative mobility of the node

- The 'inter-MANET traffic load' via each IGW, which is represented as the number of registered MANET nodes sending/receiving traffic to/from Internet.

- The 'intra-MANET traffic load' within the network topology managed by each IGW, which is related to the optimal node density to deliver traffic successfully.

- The residual energy at the IGW.

\subsubsection{Relative Mobility Criteria}

The mobility is computed depending on the power level sensed at the receiving node $\left(P_{r x}\right)$.

- During the ideal environment, Friis free space propagation model is utilized that exploits an inverse-square dependence of the ratio of received and transmit power on the physical distance among the transmitter and the receiver.

$$
\text { i.e. } \frac{P_{r x}}{P_{t x}} \propto \frac{1}{d^{2}} .
$$

- During the real environment, the computation of the distance among the transmitter and receiver from the calculated signal strength may not be feasible due to complications concerned with exact channel modeling.

- From the ratio of $P_{r x}$ among the two consecutive packet transmissions from a neighbor node, the information concerned with relative mobility among the two nodes can be acquired. Through this information, the relative mobility metric $R M_{Y}(X)$ at a node $\mathrm{j}$ with respect to $\mathrm{i}$ is calculated as follows.

$$
R M_{j}(i)=10 \log _{10} \frac{P_{r x_{i \rightarrow j}}^{\text {new }}}{P_{r x_{i} \rightarrow j}^{\text {old }}}
$$

From the above relative mobility equation, two cases are analyzed.

\section{Case 1}

If $P_{r x_{i} \rightarrow j}^{\text {new }}<P_{r x_{i} \rightarrow j}$ old

Then

$R M_{j}$ (i) is negative which specifies that the nodes are moving away from each other.

End if

Case 2

If $P_{r x_{i} \rightarrow j}^{\text {new }}<P_{r x_{i} \rightarrow j}^{\text {old }}$ Then $R M_{j}(\mathrm{i})$ is positive which indicates that the nodes are moving closer to each other.

End if

\subsubsection{Inter and Intra MANET Traffic Load}

$T L_{\text {inter }}$ is the inter-MANET traffic load which is given by the number of current registered MANET nodes $\beta_{\operatorname{Re} g}(j)$ at $I G W_{j}$ that require Internet connectivity.

$$
T L_{\text {inter }}(j)=\beta_{\text {reg }}(j)
$$

$T L_{i n t r d}(i, j)$ is the intra-MANET traffic load in the network topology $\left(X_{j}, Y_{j}\right)$ managed by $I G W_{j}$. It is determined based on the optimal node density $\rho$, and the average node degree AvgNd. [14]

$$
T L_{\text {int } r a}(i, j)=\left\{\frac{1}{A v g N d}\right\}
$$

where $A v g N d=\rho \pi r^{2}$

\subsubsection{Weight Calculation}

Let $\alpha, \beta, \gamma$ and $\lambda$ be the metric denoting mobility, interMANET traffic load, intra MANET traffic load, and residual energy respectively.

Let $S_{i j}$ represent the value of each metric.

Let $G W_{j}$ be the gateway node candidate

Let $n_{i j}$ be the normalized value of the metric whose range is [0, 1]

Let $q_{i}$ be weighting factor

Let $r$ be the number of metrics

Let $q_{\alpha}, q_{\beta}, q_{\gamma}$ and $q_{\lambda}$ be the weighing factors of mobility, inter and intra MANET traffic load, and remaining energy respectively.

The metric values are represented by the matrix $Z_{i j}$ in (using eq. 4).

$$
\begin{gathered}
\alpha \\
G W_{1} \\
G W_{2} \\
\cdot \\
\cdot \\
\cdot \\
G W_{i}
\end{gathered}\left[\begin{array}{cccc}
s_{11} & s_{12} & s_{13} & s_{14} \\
s_{21} & s_{22} & s_{23} & s_{24} \\
\cdot & \cdot & \cdot & \cdot \\
\cdot & \cdot & \cdot & \cdot \\
s_{i 1} & s_{i 2} & s_{i 3} & s_{i 4}
\end{array}\right]
$$

As every individual metrics has different ranges and value units, there is a necessity to scale the metrics to acquire all metric values in a non-dimensional form. This scaling technique involves the two categories which are as follows.

1) Positive criteria

2) Negative criteria

Our approach involves computation of the residual energy with the positive criteria since it is superior to have higher energy value. The positive criterion is represented using the following formula.

$$
n_{i j}=\frac{s_{i j}-s_{j}^{\min }}{s_{j}^{\max }-s_{j}^{\min }}
$$

Consequently the mobility metric and traffic loads are computed using the negative criteria as these values will be required in the lower range. The negative criterion is represented using the following formula. 


$$
n_{i j}=\frac{s_{j}^{\max }-s_{i j}}{s_{j}^{\max }-s_{j}^{\min }}
$$

Following the normalization of the every metric to the equivalent scale, user weighting factor is defined that denotes the priority in comparison to the consequence of metrics. The direct specification methodology is used to determine the weighting factors. The assignment of the weighting factor is more adaptable and each node can choose weighting factor as per priority.

The condition for the assignment of weighting factor is as follows.

$$
\sum_{j=1}^{r} q_{j}=1
$$

where $q_{j}=\left\{q_{\alpha}, q_{\beta}, q_{\gamma}\right)$ and $\left.q_{\lambda}\right\}$

The overall weight value $(\mathrm{Q})$ for each gateway candidate is computed by multiplying the weighting factors and normalized metric values and summing all these value using the following formula.

$$
Q=\sum_{j=1}^{r} q_{j} n_{i j}
$$

\subsubsection{Gateway Selection}

The network model has multiple internet gateways IGWs $[\mathrm{IGW} 1, \mathrm{IGW} 2, \ldots, \mathrm{IGWn}]$ in a foreign MANET domain, and each $I G W_{j}$ manages a network topology $\left(X_{j}, Y_{j}\right)$, which can be overlapped with those managed by other IGWs.

- Each $\mathrm{IGW}_{\mathrm{j}}$ attaches to its RREP the following information

$$
\left\{X_{j}, Y_{j}, \beta_{\operatorname{Re} g}(j), T_{j}, E(j)\right\}
$$

where $\left(X_{j}, Y_{j}\right)$ is the managed topology size of $I G W_{j}$

$\beta \operatorname{Re} g(j)$ is the number of registered MANET nodes with $I G W_{j}$ for the inbound/outbound traffic from/to the Internet.

$T_{j}$ is the total MANET nodes in the managed topology of $I G W_{j}$.

$\mathrm{E}(\mathrm{j})$ is the residual energy of $I G W_{j}$.

- This RREP is sent directly to the source MANET upon receiving its RREQ.

- Each $I G W_{j}$ determines $\left[\beta_{\operatorname{Re} g}(j), T_{j}, E(j)\right]$ by the periodic hello packet exchange of the neighbor discovery process, or by the on-demand RREQ/RREP packet exchange of the route discovery process.

- Whenever a visited or a local MANET node, which requires the Internet connectivity, receives RREP from multiple $I G W_{S}$ in the same MANET domain, the IGW with highest weight value is chosen using the section 3.3.3.

- $I G W_{j}$ does not know the existence of a visiting MANET node $\mathrm{i}$ in its managed network topology until a registration occurs. And so the average node degree is different from a local MANET node and a visiting MANET node.

- Each MANET node i, upon requesting Internet connectivity, register to one of the IGWs discovered.

\section{SIMULATION RESULTS 4.1 Simulation Setup}

We evaluate our efficient multi-criteria gateway selection based multi-path DYMO extension (MCS-DYMO) protocol through NS2 [19] simulation. We use ns2 version 2.28 with DYMO extension. We consider a hybrid network deployed in an area of 1200 X $1200 \mathrm{~m}$. There are 15 mobile nodes in the MANET domain. There are 5 gateway nodes connected with a fixed internet host through a router (ref. figure 3).

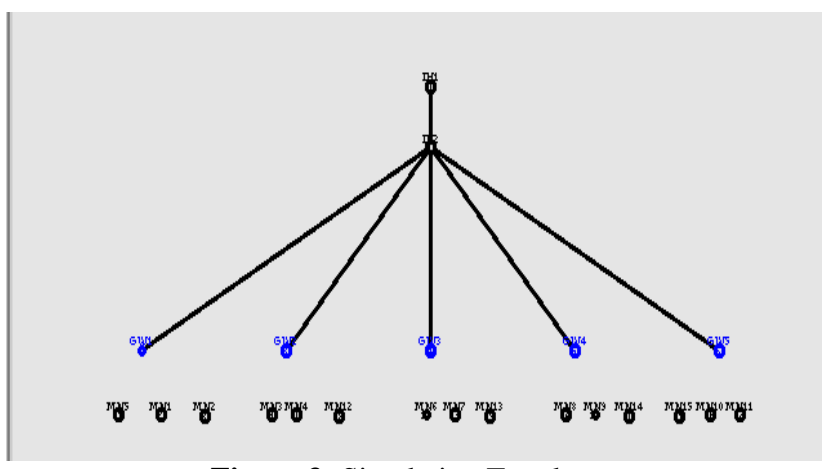

Figure 3: Simulation Topology

The simulated traffics are CBR and FTP. We have varied the traffic flows to increase the traffic load in the network. The following table summarizes the simulation parameters used.

Table 1. Simulation Settings

\begin{tabular}{|l|l|}
\hline Mobile Nodes & 15 \\
\hline MAC protocol & 802.11 \\
\hline Propagation Model & TwoRayGround \\
\hline Area Size & 1200 X 1200 \\
\hline Simulation Time & 50 seconds \\
\hline Radio Range & $250 \mathrm{~m}$ \\
\hline Wired Nodes & 2 \\
\hline Gateway nodes & 5 \\
\hline Traffic Source & CBR and TCP \\
\hline Packet Size & 512 \\
\hline Data Rate & $250 \mathrm{~Kb}$ \\
\hline Mobility Model & Random Way Point \\
\hline Speed & $5 \mathrm{~m} / \mathrm{s}$ to $25 \mathrm{~m} / \mathrm{s}$ \\
\hline Initial Energy & $5.1 \mathrm{~J}$ \\
\hline Transmit Power & 0.66 Watts \\
\hline Receiving Power & 0.0695 Watts \\
\hline Idle Power & 0.035 Watts \\
\hline Traffic Flows & $1,2,3,4 \& 5$ \\
\hline
\end{tabular}

\section{2. Performance Metrics}

We evaluate mainly the performance according to the following metrics.

Average end-to-end delay: The end-to-end-delay is averaged over all surviving data packets from the sources to the destinations.

Energy Consumption: It is the average energy consumption of all nodes in sending, receiving and forward operations

Average Packet Delivery Ratio: It is the ratio of the number .of packets received successfully and the total number of packets transmitted.

Drop: It is the average number of packets dropped during the transmission.

We compare our MCG-DYMO protocol with the normal DYMO protocol. The simulation results are given in the next section. 


\subsection{Results}

A. Based on Number of Flows

In the first experiment, we vary the number of CBR traffic flows from 1 to 5 . We randomly select some mobile nodes as sources to get data from the internet through the gateway nodes. The nodes are set to move with a fixed speed of $25 \mathrm{~m} / \mathrm{s}$.

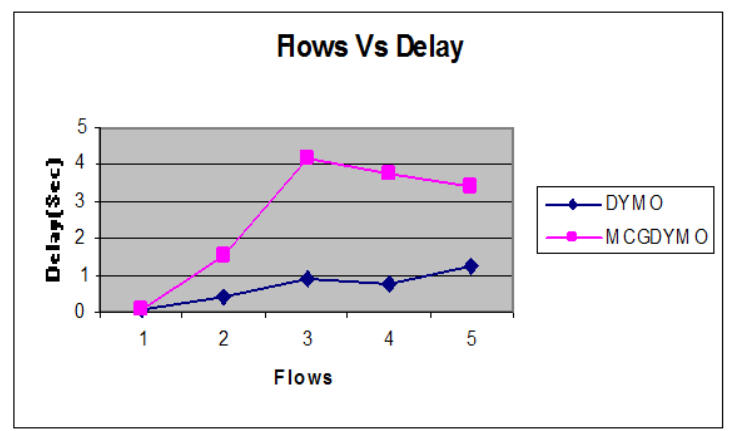

Figure 4: Flows Vs Delay

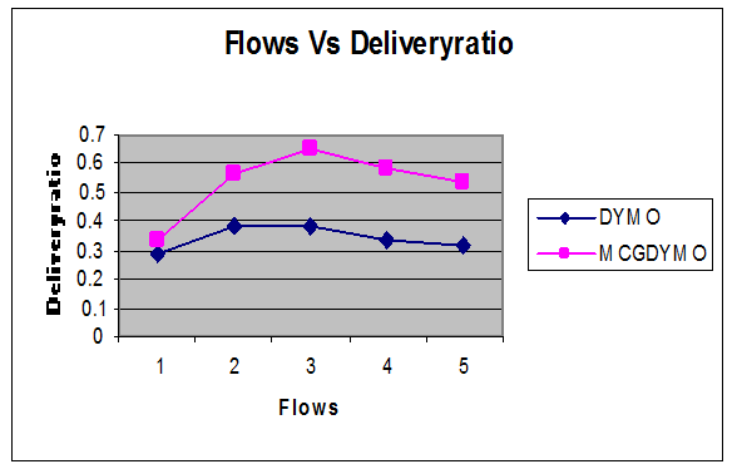

Figure 5: Flows Vs Delivery ratio

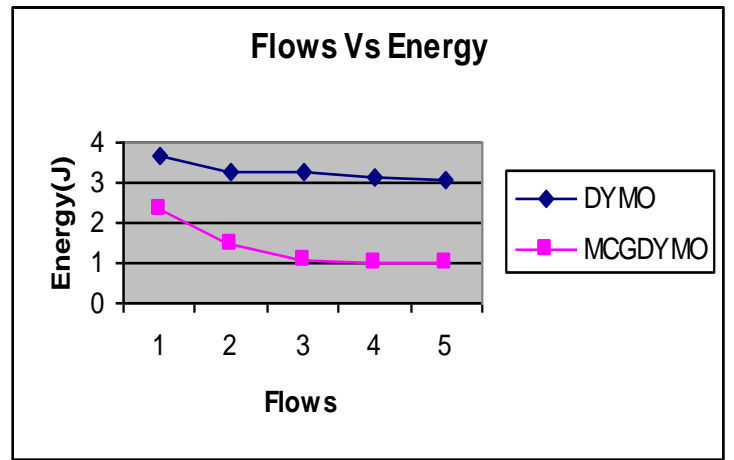

Figure 6: Flows Vs Energy

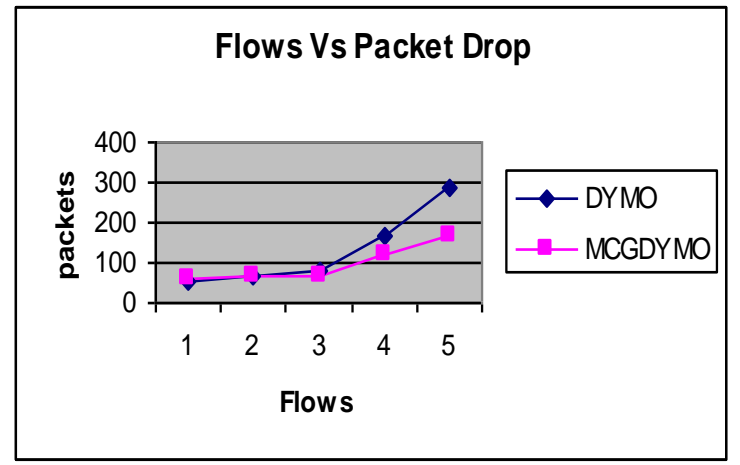

Figure 7: Flows Vs Drop

Since residual energy is considered as one of the metrics for selecting the IGW, the energy consumption in MCGDYMO is less when compared with the normal DYMO protocol. We can observe this from Figure 6 for TCP traffic.Since traffic load is considered in the gateway selection, the packet delivery ratio for the proposed MCGDYMO protocol is significantly more when compared with DYMO. Figure 5 shows the result of packet delivery ratio for CBR traffic flows. For the same reason, the end to end delay is also reduced in the MCGDYMO protocol, which can be observed from the figure 4. Since MCGDYMO involves multiple paths, the packet drop is very much reduced. Figure 7 shows that the packet drop is less in MCGDYMO when compared to DYMO.

\section{B. Based on Node Speed}

In the second experiment, we vary mobile speed from $5 \mathrm{~m} / \mathrm{s}$ to $25 \mathrm{~m} / \mathrm{s}$ for the 5 CBR traffic flows.

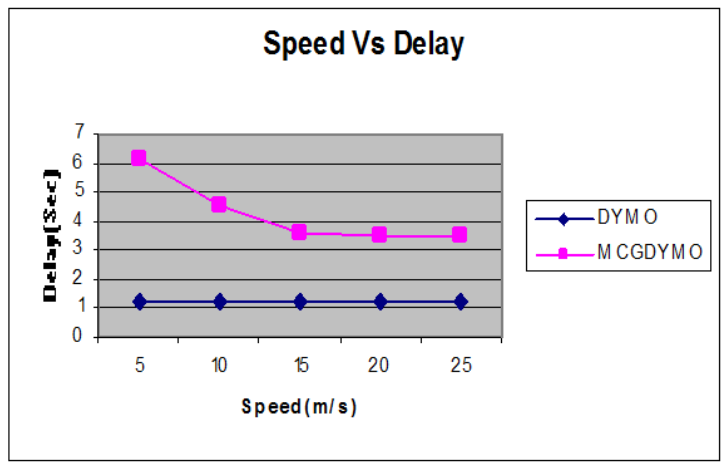

Figure 8: Speed Vs Delay

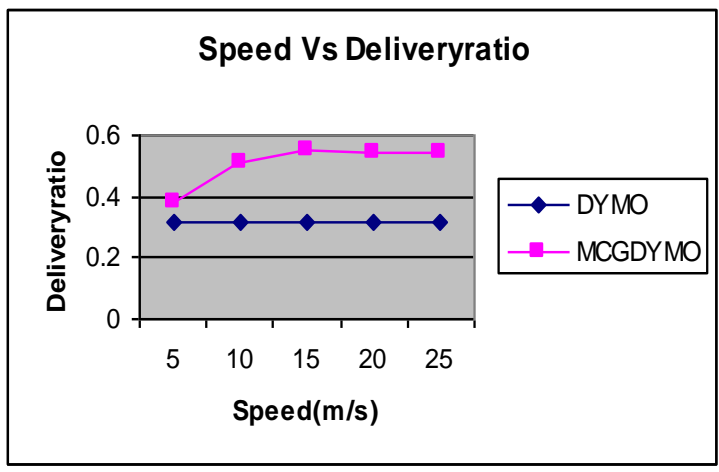

Figure 9: Speed Vs Delivery ratio

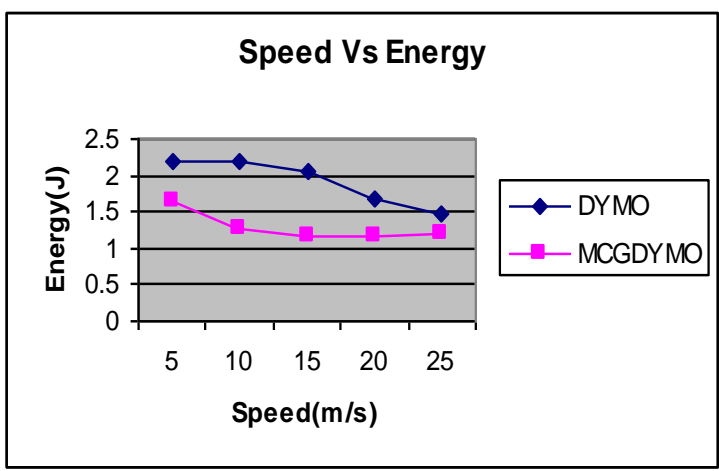

Figure 10: Speed Vs Energy 


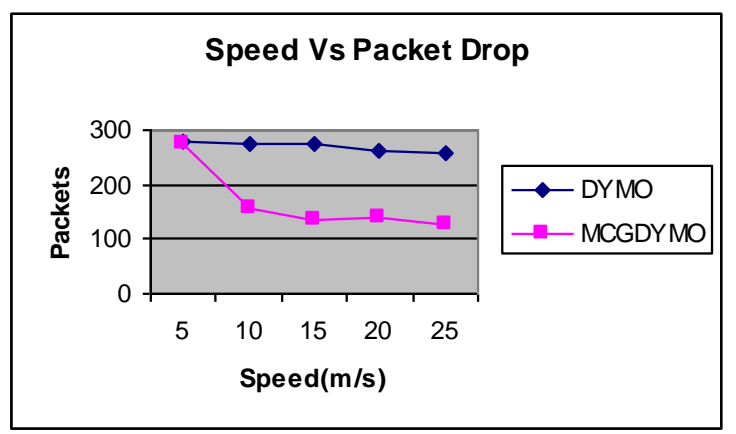

Figure 11: Speed Vs Drop

From the figures $8,9,10$ and 11 , we can see that MCG-DYMO outperforms DYMO in terms of delay, packet delivery ratio, energy and packet drop, respectively.

\section{CONCLUSION}

In this paper, we have proposed a multi-criteria gateway selection and multipath routing protocol for Hybrid MANETs. The multipath routing is established using the dynamic MANET on-demand (DYMO) routing protocol. For gateway selection, a combined weight value is determined based on the metrics mobility, inter and intra MANET traffic load and residual energy using simple additive weighing (SAW) technique. As the scaling approach, this technique uses the positive and negative criteria so the metrics turns out to be a non-dimensional value. Following the normalization of the every metric to the equivalent scale, the weighting factor is determined using direct specification methodology. Then the overall weight value for each gateway candidate is computed by multiplying the weighting factors and normalized metric values and further summing of all these value. Among the selected path from the multiple paths, the gateway with maximum weight is selected. If such a gateway does not exist, alternate path is selected from the multi path set. By simulation results, we have shown that the proposed approach is a efficient technique for gateway selection.

\section{REFERENCE}

[1] Mamoun Hussein Mamoun, "A Novel Routing Algorithm for MANET" International Journal of Electrical \& Computer Sciences 2010

[2] Jun-Zhao Sun "Mobile Ad Hoc Networking: An Essential Technology for Pervasive Computing" Proceedings of International Conferences on info-tech and Info-net 2001.

[3] Takeshi Matsuda · Hidehisa Nakayama, Xuemin (Sherman) Shen · Yoshiaki Nemoto, Nei Kato "Gateway Selection Protocol in Hybrid MANET Using DYMO Routing" Springer -Mobile networks and applications, May 2009.

[4] Yu-chee tseng, Wen-hua liao, Shih-lin Wu "Mobile Ad Hoc Networks and Routing Protocols" Handbook of Wireless Networks and Mobile Computing, 2002.

[5] Velmurugan Ayyadurail and Rajaram Ramasamy "Internet Connectivity for Mobile Ad Hoc Networks Using Hybrid Adaptive Mobile Agent Protocol" The International Arab Journal of Information Technology, Vol. 5, No. 1, January 2008.

[6] Kristian L. Espensen, Mads K. Kjeldsen, and Lars M. Kristensen "Towards Modelling and Validation of the DYMO Routing Protocol for Mobile Ad-hoc Networks" Springer- Petri nets 2008.
[7] Junho Chung, Yonghwan Kwon, Bosung Kim, Hakkwan Kim, Kyungmin Lee, Dowon Hyun and Juwook Jang "A Route Recovery Scheme in DYMO for Efficient Ad-hoc" International Conference on Ubiquitous City Technology, Jan. 2008.

[8] Kamaljit I. Lakhtaria and Prof. Bhaskar N. Patel "Comparing Different Gateway Discovery Mechanism for Connectivity of Internet \& MANET" International Journal of Wireless Communication and Simulation .2010.

[9] Dong-Won Kum, Jin-Su Park, You-Ze Cho, and ByoungYoon Cheon "Performance Evaluation of AODV and DYMO Routing Protocols in MANET" IEEE 2010.

[10] A.Boomarani Malany, V.R.Sarma Dhulipala, and RM.Chandrasekaran "Throughput and Delay Comparison of MANET Routing Protocols" ICSRS Publication, 2009.

[11] Quan Le-Trung, Paal E. Engelstad, Telenor R\&I, Tor Skeie, Simula, Frank Eliassen IFI/UiO, " Internetworking Mobile Ad-Hoc Networks with the Internet", Master Thesis topic Ref. no"2009.Spring.Master.02".

[12] Rakesh Kumar, Manoj Misra and Anil K. Sarje, "A Proactive Load-Aware Gateway Discovery in Ad Hoc Networks for Internet Connectivity", International Journal of Computer Networks \& Communications (IJCNC) Vol.2, No.5, September 2010

[13] P. Venkateswaran and Pritam Som, Rahul Ghosh, Aritra Das, S. Hansda, R. Nandi, " SmartGate: A MAC Protocol for Gateway Discovery in Clustered Ad-Hoc Networks", JOURNAL OF NETWORKS, VOL. 4, NO. 3, MAY 2009

[14] Quan Le-Trung, Paal E. Engelstad, Tor Skeie, and Amirhosein Taherkordi, " Load-Balance of Intra/InterMANET Traffic over Multiple Internet Gateways", Proceeding of the 6th International Conference on Advances in Mobile Computing and Multimedia (MoMM), 2008.

[15] Amandeep Kaur Saggu, Parminder Singh and Sandeep Singh Kang, " Providing Internet Connectivity and QOS of Reactive and Proactive Protocols Using Gateway Discovery in Mobile Ad Hoc Networks", International Journal of Computer Science and Telecommunications, Volume 2, Issue 6, September 2011.

[16] Khaleel Ur Rahman Khan, Prof. A Venugopal Reddy, Rafi U Zaman, " An Efficient Integrated Routing Protocol for Interconnecting Mobile Ad Hoc Network and the Internet", International Journal of Computer and Electrical Engineering, Vol. 1, No. 1, April 2009.

[17] Quan Le-Trung, Paal E. Engelstad, Vinh Pham, “ Providing Internet connectivity and mobility management for MANETs", International Journal of Web Information Systems, pp.239 - 263, Vol. 5 Issue: 2, 2009.

[18] Dr Yogesh Chaba, Dr R B Patel, Rajesh Gargi, “ Efficient Multipath DYMO Routing Protocol with Gateway Selection for Hybrid MANETs", International Journal of Computer Theory and Engineering, Vol,4,No 4,2012

[19] Network Simulator, http://www.isi.edu/nsnam/ns 


\section{AUTHORS PROFILE}

Rajesh Gargi received the B.Tech. degree from Regional Engineering College, Kurukshetra. He obtained his M.Tech degree in Computer Sc. \& Engg from Guru Jambheshwar University of Science \& Technology, Hisar and perusing PhD from the same University. He is working as Associate Professor in Computer Sc. \& Engg, Department at Indus Institute of Engineering and Technology Kinana, Jind. His Research areas are Computer Networks and Mobile Communication. He has published more than 10 papers in national, international journals and conferences of repute.

Dr Yogesh Chaba received the B.E. degree in Computer Sc. \& Engg with DISTINCTION from Marathwada University, Aurangabad in year 1993. He obtained his MS degree in Software Systems from BITS Pilani and PhD degree from Guru Jambheshwar University of Science \& Technology, HISAR. He is working as Associate Professor in Deptt of Computer Sc. \& Engg, Guru Jambheshwar University of Science \& Technology, HISAR. He worked as Chairman, Deptt of Computer Sc. \& Engg, Guru Jambheshwar University of Science \& Technology, HISAR for three years. His Research areas are Computer Networks and mobile communication. He has published more then 75 papers in national and international journals and conferences of repute including IEEE, Springer and Science Direct Journals. He is Principal Investigator of two major research projects funded by All India Council for Technical Education and University Grants Commission, INDIA in the area of Network Security and Ubiquitous. He is also Deputy Coordinator of SAP project funded by University Grant Commission. He has vast international exposure as he has visited different universities and research institutions in USA, UK and China for academic assignments. He is also recipient of "Young Scientist Award" by International Academy of Physical Sciences for year 2002.

Dr. R. B. Patel ,Dean ,Faculty of Information Technology \& Computer Science ,Deenbandhu Chhotu Ram University of Science \& Technology, Murthal. He received $\mathrm{PhD}$ from IIT Roorkee in Computer Science \& Engineering, PDF from Highest Institute of Education, Science \& Technology (HIEST), Athens, Greece, MS (Software Systems) from BITS, Pilani and B. E. in Computer Engineering from M. M. M. Engineering College, Gorakhpur, UP. He has two patents, numerous best paper awards and more than 100 publications to his credit. He is member of bodies like IEEE, ISTE. 